Sportis. Revista Técnico-Científica del Deporte Escolar, Educación Física y Psicomotricidad

Sportis. Scientific Technical Journal of School Sport, Physical Education and Psychomotricity

Artículo Original. Fomento de estilos de vida activos en la escuela: práctica de actividad física, edad y género. Vol. V, nº 1; p. 53-69, enero 2019. A Coruña. España ISSN 2386-8333
\end{abstract}

\title{
Fomento de estilos de vida activos en la escuela: práctica de actividad física, edad y género
}

\section{Active lifestyles development at school: physical activity, age and gender}

\author{
Cantero Castrillo, Pedro; Mayor Villalaín, Alba; Toja Reboredo, Belén; González Valeiro, Miguel \\ Grupo de investigación de la Universidad de A Coruña en Educación, Salud y Actividad Física: \\ Estudios de género
}

Autor de correspondencia: Pedro Cantero cantero_46@hotmail.com

Cronograma editorial: Artículo recibido: 24/07/2018 Aceptado: 13/11/2018 Publicado: 01/01/2019

DOI: https://doi.org/10.17979/sportis.2019.5.1.3680

\section{Resumen}

La alta inactividad física que presentan los jóvenes escolares hace necesaria la realización de estudios que busquen conocer los motivos de esta situación y busquen propuestas para su mejora. El objetivo de este estudio fue analizar las características que definen a escolares de secundaria de centros que disponen de un programa de fomento de actividad física (AF) (Proyecto Deportivo de Centro) de la Comunidad Autónoma de Galicia en función del índice de AF, el género y la edad. La muestra está compuesta por 1697 sujetos (824 niños y 873 niñas) y 2335 padres/madres. Como instrumento de recogida de datos se utilizó el "Cuestionario Internacional sobre el Estilo de Vida de los Alumnos". Los resultados sugieren que un 66,9\% de los alumnos tiene un índice de actividad poco activo o muy poco activo (56\% niños y 77,3\% niñas) y un índice de actividad activo o muy activo de un 33,1\% (44\% niños y 22,7\% niñas). Este estudio presenta evidencias de que existen diferencias de género y de edad a la hora de cuantificar la cantidad de horas de práctica de actividad físico-deportiva, al igual que se observa una disociación entre la percepción de práctica y lo realizado realmente. También se ve una relación significativa entre la práctica de AF y el género, no encontrándose lo mismo al analizar el curso académico. A modo conclusión, se observa una elevada inactividad física, sobretodo en el género femenino, junto con un desconocimiento de esta situación, tanto por parte de los alumnos y alumnas, como de sus padres y madres.

\section{Palabras clave}

Género; Percepción de práctica; Actividad física; Edad.

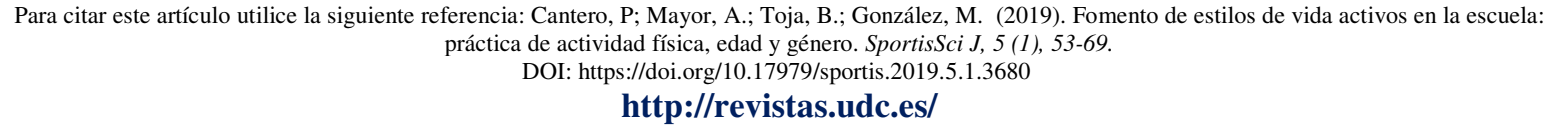




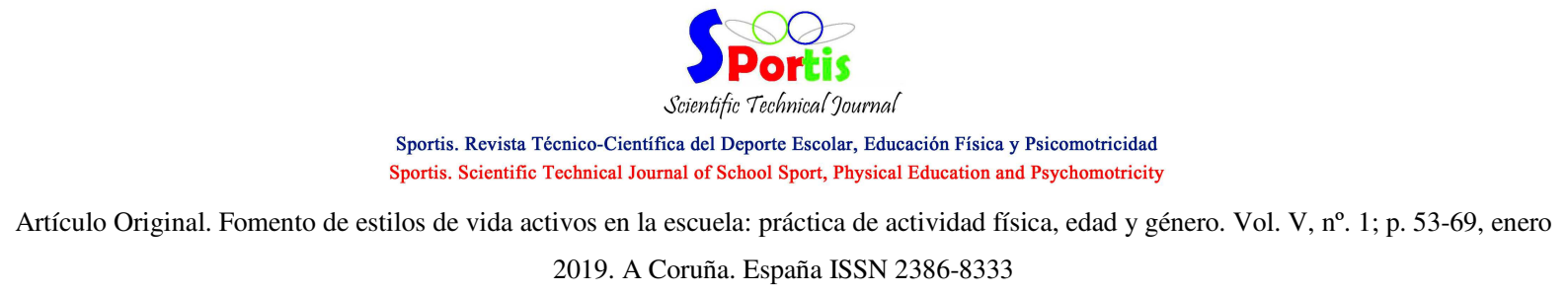

Abstract

The high physical inactivity that young students present makes it necessary to carry out studies that seek to know the reasons for this situation and seek proposals for its improvement. The aim of this study was to analyze the characteristics that define high school students of a schools with a program (Sport Project Centre) to promote physical activity in Galicia in terms of physical activity, gender and age rate. The sample is composed of 1697 subjects ( 824 boys and 873 girls) and 2335 parents. "The International Questionnaire about Lifestyles in Students" was used as an instrument of data collection. The results suggest that $66.9 \%$ of students have a low or very low rate of physical activity (56\% boys and $77.3 \%$ girls) and $33,1 \%$ as active or very active ( $44 \%$ boys and $22.7 \%$ girls). This study provides evidence that there are gender and age differences when when quantifying the number of hours of physical activity. In addition, there is a dissociation between the practice perception and what is actually realized. There is also a significant relationship between the practice of physical activity and gender, not being the same when analyzing the academic course.In conclusion, there is a high physical inactivity, especially in females, together with a lack of knowledge of this situation, both from the students and their parents.

\section{Keywords}

Gender; Practice perception; physical activity; Age.

\section{Introducción}

La inactividad física está comenzando a ser un gran problema en los países desarrollados, esto se debe al aumento de comportamientos sedentarios, como ver la televisión o el uso de internet, y a la evasión de tareas que impliquen un esfuerzo físico (Ramos, Rivera, Moreno \& Jiménez-Iglesias, 2012). Consecuentemente, de esta inactividad física derivan afecciones y enfermedades que se están incrementando en la sociedad, como son la obesidad, diabetes, enfermedades coronarias e hipertensión, entre otras (Organización Mundial de la Salud, 2010). Tampoco debemos olvidarnos de las consecuencias que tienen la inactividad física en la economía de los países que presentan estilos de vida poco activos y altas tasas de morbilidad. Según ISCA (2015) la inactividad física genera un gasto económico en salud de 990 millones de euros anuales en España. Esta situación genera una gran preocupación, dado el conocimiento existente del impacto positivo que tienen los estilos de vida activos sobre la salud, tanto a nivel físico, psicológico, social (Ekelund et al., 2012) y económico (ISCA, 2015).

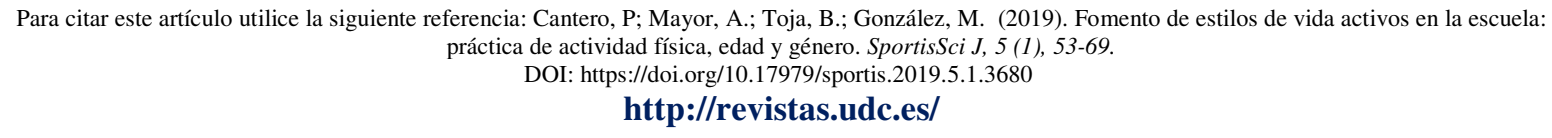




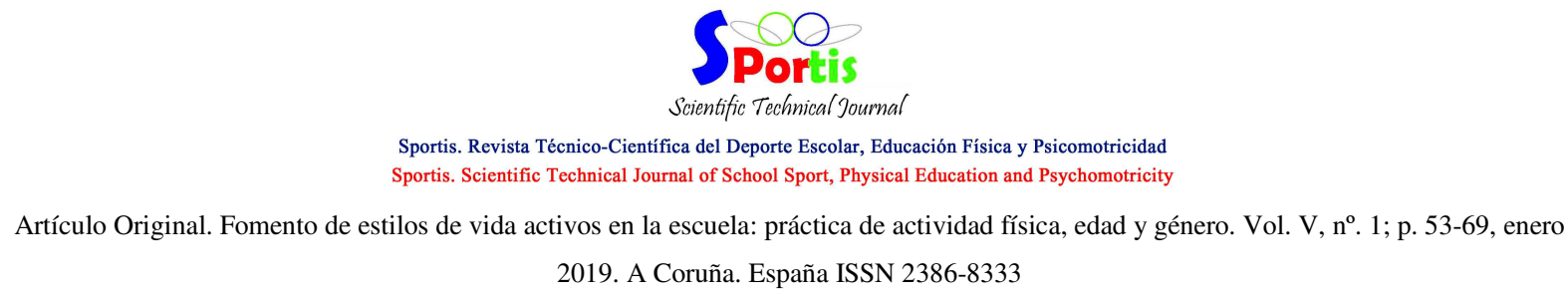

Se evidencia una diferenciación en la cantidad de práctica de AF en función del género, siendo el género femenino el que menos práctica de AF realiza, tanto en jóvenes (Cocca et al., 2015; ISCA, 2015; Oviedo et al., 2013) como en adultos (ISCA, 2015). Para explicar estas diferencias existen diferentes teorías, desde la orientación de las propuestas de AF que existen en la actualidad, pudiendo estas presentar una carga deportiva que resulta más atractiva a los niños que a las niñas (Oviedo et al., 2013), hasta una explicación sociológica, donde los niños suelen ser más propensos a practicar ya que tienen más oportunidades fuera de la escuela y presentan experiencias más positivas que las niñas (Mota \& Sallis, 2002; Wold \& Anderssen, 1992). Es por ello que será necesario realizar nuevas propuestas y planteamientos pedagógicos a fin de lograr que las mujeres incrementen el tiempo de práctica de AF (Oviedo et al., 2013).

A nivel de edad, se observa un descenso de la práctica de $\mathrm{AF}$, en ambos géneros, en función del aumento de los años (Currie et al., 2012). Estos resultados vienen reforzados por estudios que muestran como los alumnos de primaria son más activos que los de secundaria (Arias, 2014) y estos a su vez practican mayor cantidad de AF que los universitarios (Cocca et al,. 2015). Siendo la adolescencia un momento especialmente importante donde la práctica de AF suele reducirse y los hábitos alimenticios se ven afectados negativamente (Sallis et al., 2016; Serra \& Aranceta, 2000)

Observando los problemas que derivan de unos comportamientos sedentarios se puede afirmar la importancia que tiene mantener una vida activa. Para lograr este cambio en la sociedad es necesario inculcar unos buenos hábitos de vida a los jóvenes, ya que, es a lo largo de la infancia y la adolescencia cuando se instauran muchas de las pautas de comportamiento que van a tener una influencia poderosa sobre la salud en la vida adulta (Janz, Dawson \& Mahoney, 2000).

Para aumentar la práctica de AF en niños y adolescentes y crear buenos hábitos hay que tener en cuenta la escuela como el entorno más apropiado para ello (Kahn et al., 2002), siendo la Educación física el elemento al que se le brinda una oportunidad especial al tener el alumno que asistir a clase a diario, y, al ser la Educación Física una asignatura atrayente (Campbell, Crews \& Sinclair, 2002). Además la Educación Física resulta un área clave para el

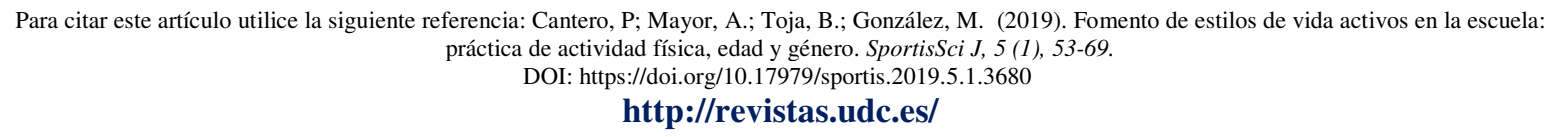




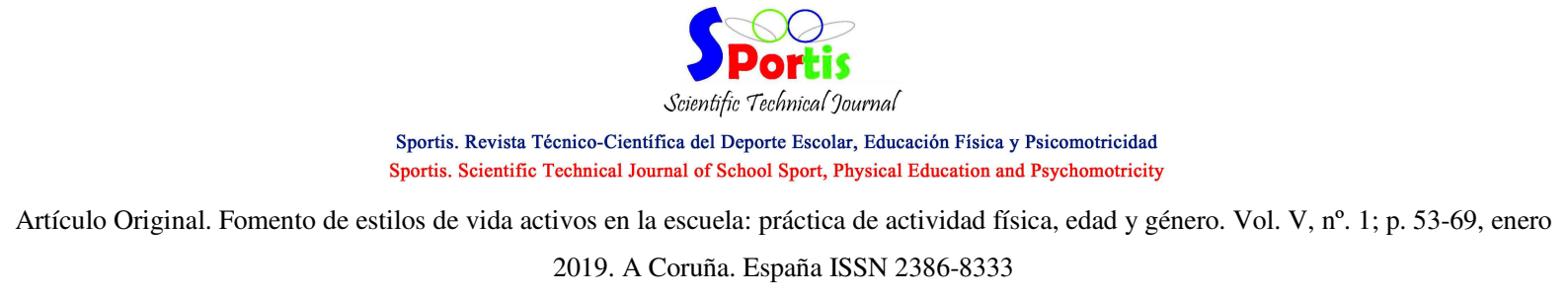

cumplimiento de las recomendaciones de A.F. en la edad adolescente, tanto por el tiempo de A.F. que puede proporcionar (Fairclough \& Stratton, 2005), aunque siendo este insuficiente y debiéndose aumentar, como por el hecho de que sus objetivos educativos se centran en la adquisición de los conocimientos y habilidades necesarias para la adopción de un estilo de vida activo (Ahrabi-Fard \& Matvienko, 2005). También se debe destacar el papel clave que tiene la Educación Física en la promoción de A.F. entre los jóvenes, que pese a las dificultades que presenta en su ejecución, compensa si se consideran los beneficios para la salud que se derivan de tener una población de escolares físicamente activos (Camacho, Fernández, Ramírez \& Blández, 2012).

Para evitar las consecuencias derivadas de la inactividad física y el sedentarismo, la Organización Mundial de la Salud (2010) ha elaborado unas recomendaciones de AF para distintas etapas de edad. Para los jóvenes de 5-17 años, edad que recoge este estudio, se recomienda realizar como mínimo 60 minutos diarios de actividades físicas moderadas o vigorosas, incluyendo un mínimo de dos sesiones semanales de actividades que mejoren el componente óseo, la fuerza muscular y la flexibilidad (American College of Sports Medicine, 2018; American Heart Association, 2016). En la actualidad, en la Comunidad Autónoma de Galicia, se diseñó un programa denominado Plan Proxecta (Xunta de Galicia, 2013). Esta iniciativa de innovación educativa presenta en el ámbito de la promoción de AF en el centro educativo 5 subprogramas específicos: Xogade, Móvete mais, Mais e mellor actividade física, DAFIS y Proyecto Deportivo de Centro, todos ellos encaminados a aumentar la cantidad de práctica de AF de los jóvenes escolares a través de diferentes estrategias.

El objetivo de este estudio es conocer la situación actual de la población escolar gallega en referencia al nivel de práctica de AF que realizan, observando las diferencias en función del género y el curso. A su vez, se intenta conocer la percepción que tienen los propios jóvenes, al igual que sus padres acerca de la práctica de AF que realizan. Cómo último objetivo se quiere observar la relación existente entre la práctica de AF y el género.

\section{Método}

Se presenta un estudio de carácter descriptivo de encuesta.

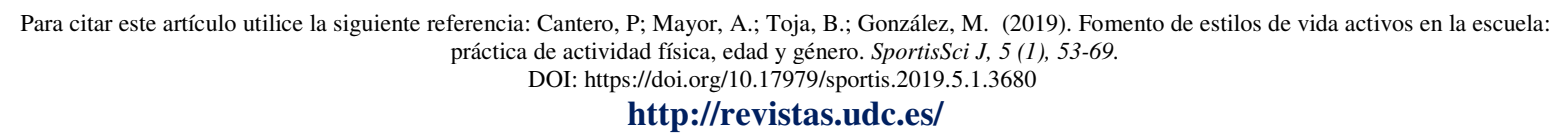


Artículo Original. Fomento de estilos de vida activos en la escuela: práctica de actividad física, edad y género. Vol. V, nº 1; p. 53-69, enero 2019. A Coruña. España ISSN 2386-8333

\section{Participantes}

La población la componen 1697 estudiantes de Educación Secundaria Obligatoria (ESO) de la Comunidad Autónoma de Galicia, 824 (48.56\%) niños y 873 (51.44\%) niñas, de $1^{\circ}, 2^{\circ}$ y $3^{\circ}$ de ESO, durante el curso académico 2013/2014.

Para establecer la muestra sólo fueron considerados los 23 centros educativos con un Proyecto Deportivo de Centro (Xunta de Galicia, 2013) debido a que nos proporciona un elemento común entre ellos, el cual es un plan para aumentar la cantidad de práctica de AF. Uno de los centros fue destinado a calcular la consistencia interna del cuestionario, dando lugar a una cifra total de 1697 alumnos y 22 centros educativos.

Se presenta en la tabla 1 la composición de la muestra distribuida por cursos académicos.

Tabla 1. Muestra de alumnos por género y curso.

\begin{tabular}{|c|c|c|c|}
\hline Género & Curso & $\mathbf{N}$ & $\%$ \\
\hline \multirow[t]{3}{*}{ Niños } & $1^{\circ} \operatorname{ESO}(*)$ & 310 & 18,26 \\
\hline & $2^{\circ} \mathrm{ESO}(* *)$ & 284 & 16,73 \\
\hline & $3^{\circ} \operatorname{ESO}(* * *)$ & 230 & 13,55 \\
\hline \multirow[t]{3}{*}{ Niñas } & $1^{\circ} \mathrm{ESO}(*)$ & 294 & 17,33 \\
\hline & $2^{\circ} \mathrm{ESO}(* *)$ & 287 & 16,92 \\
\hline & $3^{\circ} \mathrm{ESO}(* * *)$ & 292 & 17,21 \\
\hline Total & & 1697 & 100 \\
\hline
\end{tabular}

(*) $1^{\circ}$ de Educación Secundaria Obligatoria (12-13 años)

(**) $2^{\circ}$ de Educación Secundaria Obligatoria (13-14 años)

(***) $3^{\circ}$ de Educación Secundaria Obligatoria (14-15 años)

\section{Procedimiento}

La realización de la presente investigación fue autorizada por la Secretaría Xeral para o Deporte de la Xunta de Galicia. De forma previa a la recogida de datos se remitió a los centros educativos un escrito con la explicación de los objetivos del estudio y el procedimiento a seguir.

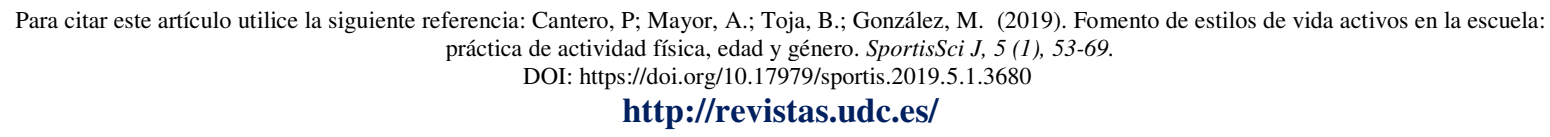


La dirección de cada colegio redirigió esta información a los padres o tutores legales solicitando la participación voluntaria de los escolares. Una vez manifestada la voluntad de participar mediante consentimiento informado por escrito, el instrumento fue administrado a los participantes en las clases de EF por los respectivos maestros. Tras la recogida de datos los cuestionarios fueron devueltos a los investigadores para su posterior tratamiento y análisis.
\end{abstract}

\title{
Medidas
}

Como instrumento de recogida de datos se utilizó el Cuestionario Internacional sobre estilo de vida de los alumnos (CIEVA) (Marques, 2010). Este cuestionario fue desarrollado a partir de las aportaciones de diversos autores. De Telama, Yang, Laakso \& Viikari (1997) se obtuvieron las preguntas referentes a los hábitos de vida; de Piéron, Telama, Naul \& Almond (1997) las de percepción de práctica y de Aaro, Wold, Kannas \& Rimpelä (1986) las de percepción de salud. A la hora de recabar información sobre estilos de vida en población escolar, estudios anteriores al nuestro, constataron la utilidad de este instrumento (Marques, 2010; Marques \& Carreiro da Costa, 2013), que posteriormente fue traducida y validada al español (Mourelle, 2014).

\section{Variables}

Las variables analizadas fueron: (1) índice de actividad físico-deportiva, constando de cuatro valores: muy poco activo, poco activo, activo y muy activo, en el caso de esta variable se tomó en cuenta a la hora de categorizarla los mínimos de práctica de AF recomendados por la Organización Mundial de la Salud (2010), siendo considerados muy poco activos (menos de 4 horas), poco activos (4-7 horas), activos (7 a 10 horas) y muy activos (más de 10 horas). (2) Género: masculino y femenino. (3) Curso de enseñanza secundaria: primero, segundo, tercero. (4) Percepción de competencia física propia: muy activo, activo, suficientemente activo, poco activo y sedentario. (5) Percepción de los padres sobre la AF que realizan sus hijos; muy activo, activo, suficientemente activo, poco activo y sedentario.

\section{Análisis estadístico}

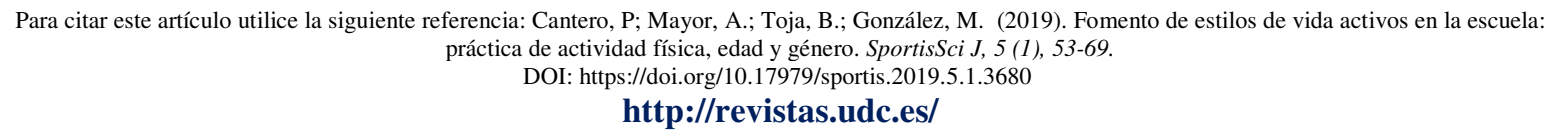


El cuestionario pasó por un grupo de expertos en metodología de investigación y para garantizar la fiabilidad de la versión final aquí empleada se procedió al cálculo del coeficiente Alpha de Cronbach obteniendo unos valores de consistencia interna aceptables (George \& Mallery, 2016) en el cuestionario de alumnos tanto a nivel global $(\alpha=.78)$ como en cada una de sus dimensiones $(\alpha>.78)$, ocurriendo igual en el de padres $(\alpha=.86)$ y en el de madres $(\alpha=$ .91). Los cuestionarios fueron recogidos en una base de datos creada con el programa SPSS Statistics 21.0 para su posterior análisis. Se realizó un análisis de frecuencias para conocer la situación real existente. Posteriormente se aplicó la prueba estadística V de Cramer para conocer la relación existente entre la práctica de AF y el género teniendo en cuenta que valores entre 0.06-0.17 indican un efecto pequeño, entre 0.18-0.29 indican un efecto medio y valores superiores a 0.30 indican un efecto grande (Cramer, 1999).

\section{Resultados}

En la tabla 2 se muestra la cantidad de AF que realizan los alumnos, divididos por género y categorizando las horas de práctica de actividad. Los datos desvelan una diferencia clara en la cantidad de horas de práctica de actividad que realizan ambos géneros, destacando el factor "muy poco activo" en las niñas con más de un $40 \%$ de las encuestadas y casi un $20 \%$ en los niños. A su vez el grupo de práctica muy activo recoge cerca de un $25 \%$ de los niños, mientras que en el caso de las niñas sólo nos encontramos con casi un $10 \%$ de niñas muy activas. El grupo de poco activos es el que más porcentaje de encuestados recoge, siendo este cercano al $35 \%$ en ambos géneros.

Tabla 2. Porcentaje AF por género

\begin{tabular}{lll}
\hline $\begin{array}{l}\text { Grado de } \\
\text { actividad }\end{array}$ & Porcentaje niños & Porcentaje niñas \\
\hline Muy poco activo & $19,6 \%$ & $42 \%$ \\
\hline Poco activo & $36,3 \%$ & $35,3 \%$ \\
\hline Activo & $20 \%$ & $13,6 \%$ \\
\hline Muy activo & $24 \%$ & $9,2 \%$ \\
\hline
\end{tabular}

Para citar este artículo utilice la siguiente referencia: Cantero, P; Mayor, A.; Toja, B.; González, M. (2019). Fomento de estilos de vida activos en la escuela: práctica de actividad física, edad y género. SportisSci J, 5 (1), 53-69. DOI: https://doi.org/10.17979/sportis.2019.5.1.3680

http://revistas.udc.es/ 
La tabla 3 muestra los porcentajes de práctica de AF por género y por curso. Los datos se caracterizan por un descenso del porcentaje de niños muy poco activos según va aumentando el curso académico, desde más de un $20 \%$ en primero a algo más del $15 \%$ en tercero. En los niños muy activos pasa totalmente lo contrario, se observa un aumento desde el $20 \%$ en primero hasta más de un $25 \%$ en tercero. En las niñas los datos no muestran diferencias entre cursos, excepto en las niñas muy poco activas, que pasan del $40 \%$ en primero a cerca del $50 \%$ en tercero.

Tabla 3. Porcentaje AF por género y curso

\begin{tabular}{|c|c|c|c|}
\hline Curso & $\begin{array}{l}\text { Grado de } \\
\text { actividad }\end{array}$ & $\begin{array}{l}\text { Porcentaje } \\
\text { niños }\end{array}$ & $\begin{array}{l}\text { Porcentaje } \\
\text { niñas }\end{array}$ \\
\hline \multirow[t]{4}{*}{$1^{\circ} \mathrm{ESO}$} & $\begin{array}{l}\text { Muy poco } \\
\text { activo }\end{array}$ & $21,4 \%$ & $40,3 \%$ \\
\hline & Poco activo & $36,9 \%$ & $36,2 \%$ \\
\hline & Activo & $20,1 \%$ & $13,7 \%$ \\
\hline & Muy activo & $21,7 \%$ & $9,9 \%$ \\
\hline \multirow[t]{4}{*}{$2^{\circ}$ ESO } & $\begin{array}{l}\text { Muy poco } \\
\text { activo }\end{array}$ & $18,7 \%$ & $39,5 \%$ \\
\hline & Poco activo & $37,5 \%$ & $37,4 \%$ \\
\hline & Activo & $18,4 \%$ & $13,9 \%$ \\
\hline & Muy activo & $25,4 \%$ & $9,3 \%$ \\
\hline \multirow[t]{4}{*}{$3^{\circ}$ ESO } & $\begin{array}{l}\text { Muy poco } \\
\text { activo }\end{array}$ & $18,4 \%$ & $46,2 \%$ \\
\hline & Poco activo & $34,2 \%$ & $32,3 \%$ \\
\hline & Activo & $21,9 \%$ & $13,2 \%$ \\
\hline & Muy activo & $25,4 \%$ & $8,3 \%$ \\
\hline
\end{tabular}

En la tabla 4 nos encontramos con el porcentaje de niños y niñas, divididos por curso, que cumplen con los requisitos mínimos de práctica de AF establecidos por la Organización

Para citar este artículo utilice la siguiente referencia: Cantero, P; Mayor, A.; Toja, B.; González, M. (2019). Fomento de estilos de vida activos en la escuela: práctica de actividad física, edad y género. SportisSci J, 5 (1), 53-69. DOI: https://doi.org/10.17979/sportis.2019.5.1.3680

http://revistas.udc.es/ 
Artículo Original. Fomento de estilos de vida activos en la escuela: práctica de actividad física, edad y género. Vol. V, nº 1; p. 53-69, enero 2019. A Coruña. España ISSN 2386-8333

Mundial de la Salud (2010), a su vez se adjuntan la percepción de práctica que tienen los alumnos, junto a cómo consideran los padres a los hijos en función de la práctica que creen que realizan.

Tabla 4. Comparativa personas que cumplen con el mínimo recomendado de AF con la percepción propia y percepción de sus padres.

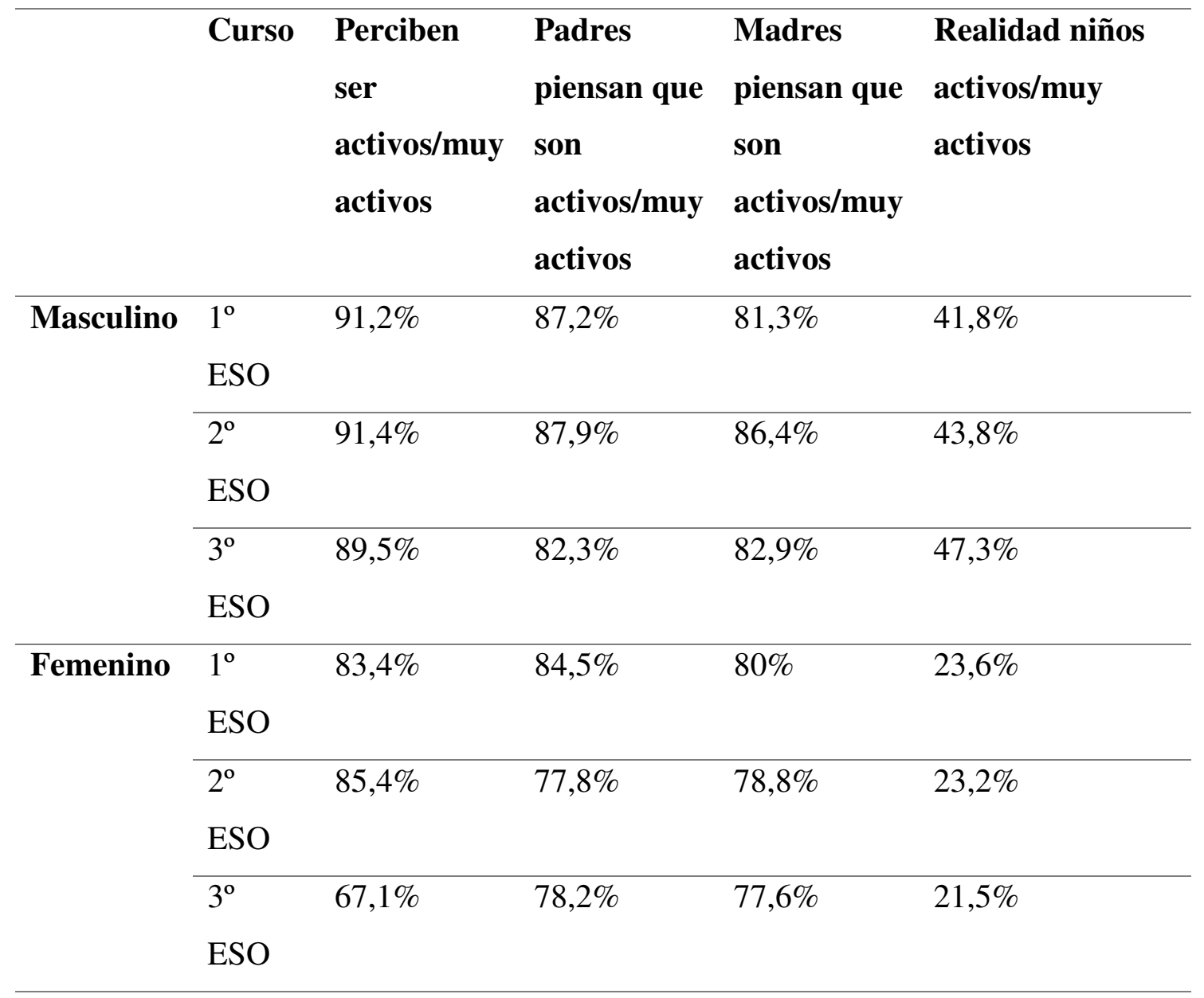

Podemos observar como el porcentaje de niños en primero de ESO que creen ser activos o muy activos y así superar los mínimos establecidos es de más de un 90\%, mientras que sus padres y madres los ven alrededor del $85 \%$, a pesar de estos datos la realidad muestra que la cantidad de niños que llegan a estos mínimos de práctica de AF supera ligeramente el $40 \%$. Estas diferencias entre realidad y percepción se van disminuyendo según va aumentando el 
curso, ya que a pesar de que los porcentajes de percepción propia de los niños siga manteniendo los mismos niveles, la cantidad de niños que superan estos mínimos de práctica de AF van aumentando progresivamente. En el caso de las niñas la cantidad de ellas que percibe ser activa o muy activa desciende considerablemente en tercero de ESO, pasando de un cercano al $85 \%$ en los primeros años a algo más de un $65 \%$ en tercero, mientras la realidad muestra que ni un $25 \%$ de ellas llegan a estos niveles. A su vez las madres que piensan que sus hijas son activas o muy activas son menores que los padres en primero de ESO, con casi un $85 \%$ de padres y un $80 \%$ de madres. En el resto de cursos los valores entre padres y madres son muy parejos, pero siempre manteniéndose en elevadas cifras.

La tabla 5 muestra los resultados acerca de la relación entre el nivel de práctica de AF y el curso académico, no encontrándose en este caso significatividad. Por el contrario, sí que se encontraron diferencias significativas entre el nivel de práctica de AF y el género. Así, se observa como los niños destacan por realizar una gran práctica de $\mathrm{AF}$, encontrándose a pocos de ellos muy poco activos. Por el contrario, las niñas siguen una relación inversa, una gran cantidad se encuentran en niveles de muy baja práctica de AF y siendo muy reducida la cantidad que son muy activas.

Tabla 5. Relación de dependencia entre la variable grado de práctica de AF y género.

\begin{tabular}{|c|c|c|c|c|c|c|c|c|c|c|c|}
\hline \multicolumn{12}{|c|}{ Práctica de AF } \\
\hline & & \multicolumn{2}{|c|}{$\begin{array}{c}\text { Muy poco } \\
\text { activo/a }\end{array}$} & \multicolumn{2}{|c|}{$\begin{array}{c}\text { Poco } \\
\text { activo/a }\end{array}$} & \multicolumn{2}{|c|}{ Activo/a } & \multicolumn{2}{|c|}{$\begin{array}{c}\text { Muy } \\
\text { activo/a }\end{array}$} & $\mathrm{P}$ & $\mathrm{V}$ \\
\hline & & $\mathrm{N}$ & $\%$ & $\mathrm{~N}$ & $\%$ & $\mathrm{~N}$ & $\%$ & $\mathrm{~N}$ & $\%$ & 0.000 & 0.273 \\
\hline \multirow[t]{2}{*}{ Género } & Niños & 161 & 19,5 & 298 & 36,2 & 164 & 19,9 & 201 & 24,4 & & \\
\hline & Niñas & 362 & 41,5 & 304 & 34,8 & 117 & 13,4 & 90 & 10,3 & & \\
\hline
\end{tabular}

Para citar este artículo utilice la siguiente referencia: Cantero, P; Mayor, A.; Toja, B.; González, M. (2019). Fomento de estilos de vida activos en la escuela: práctica de actividad física, edad y género. SportisSci J, 5 (1), 53-69. DOI: https://doi.org/10.17979/sportis.2019.5.1.3680

http://revistas.udc.es/ 


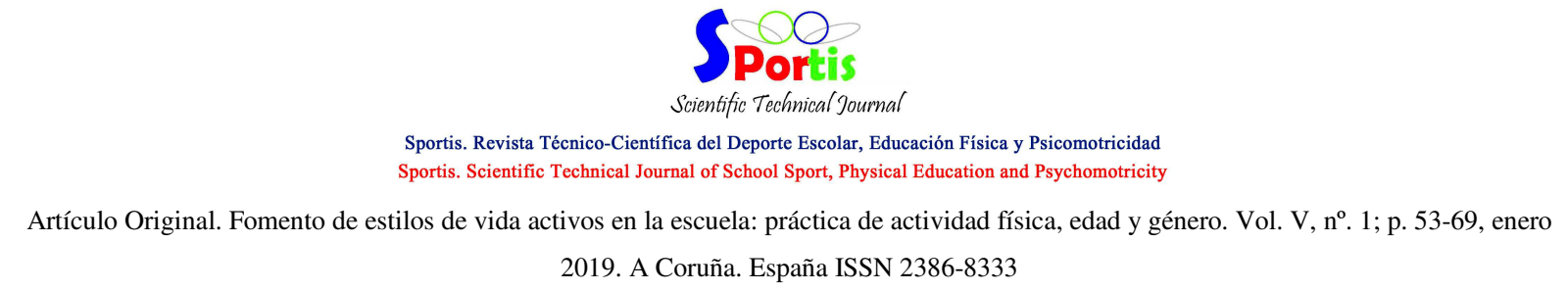

\section{Discusión}

Este trabajo proporciona información nueva sobre diferentes perfiles de percepciones y actitudes del alumnado, en relación a la práctica de AF a lo largo de la etapa de Educación Secundaria.

En general, los resultados señalan que una gran mayoría de la muestra $(66,9 \%)$ se encuentra con un índice de actividad bajo o sedentario. Estos resultados son similares a los obtenidos en otros contextos (Sallis et al., 2016), e incluso a trabajos que utilizaron el mismo procedimiento e instrumento que el presente estudio (Cantero, 2018; Marques, Martins, Carreiro da Costa \& Pièron, 2014). Dichos estudios, indican la posible incidencia negativa que esta valoración tendrá en el nivel de salud de los jóvenes estudiados, en un futuro inmediato.

Si nos centramos en las diferencias por género, observamos como las niñas se encuentran con porcentajes de práctica muy por debajo con respecto a los niños (Cantero, 2018; Moral-García, Redecillas-Peiró \& Martínez-López, 2012), a pesar de dedicar estos un mayor tiempo al uso de métodos de entretenimiento pasivos (Biddle, Gorely \& Stensel, 2004; MoralGarcía et al., 2012). Aunque también existen estudios que no encontraron diferencias entre género (Abarca-Sos, Zaragoza-Casterad, Generelo-Lanaspa \& Julián-Clemente, 2010).

El curso académico también aparenta ser un factor importante y decisivo a la hora de cuantificar la cantidad de práctica de AF que realizan los jóvenes, en nuestro estudio se puede observar un descenso de la práctica según aumenta el curso de las niñas, por el contrario, los niños sufren la tendencia contraria, viéndose aumentadas las cifras de práctica según va aumentando el curso. Diferentes estudios han analizado esta temática, estando habitualmente los trabajos más destinados a observar la edad en vez del curso, pero estando estos dos factores muy relacionados. En la bibliografía encontrada se destaca un descenso de la práctica de AF al llegar al final de la adolescencia (Moral-García et al., 2012; Sallis, 2000; Telama \& Yang, 2000). Destacar el estudio de Marques et al. (2014) que tras realizar un estudio en escuelas militares, no encontraron una reducción de la práctica de AF al aumentar la edad en jóvenes adolescentes. Esto sugiere que el contexto en el que se encuentren los jóvenes puede cambiar la tendencia de disminución de práctica (Marques et al., 2014).

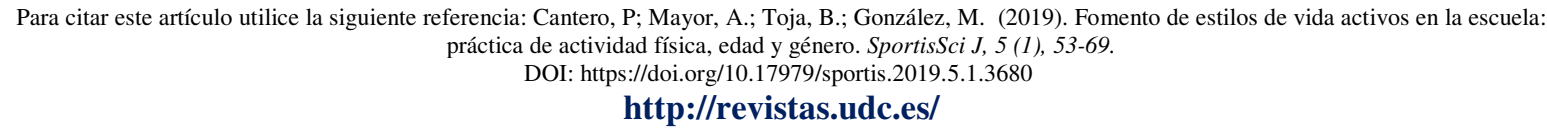
práctica de actividad física, edad y género. SportisSci J, 5 (1), 53-69. DOI: https://doi.org/10.17979/sportis.2019.5.1.3680

http://revistas.udc.es/ 


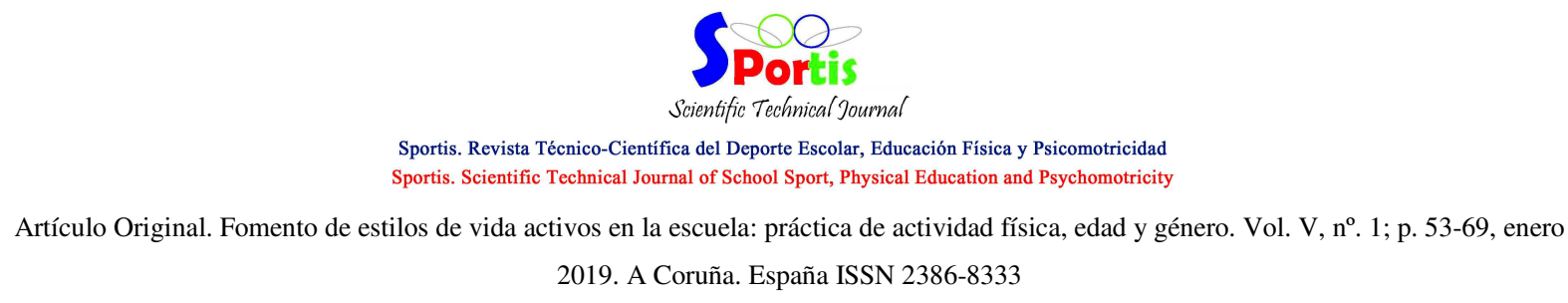

Se ha de destacar la importancia que tienen los padres y madres sobre sus hijos, ya que aportan una gran influencia en la práctica de AF de los jóvenes (Laird, Fawkner, Kelly, McNamee \& Niven, 2016; Mendonça, Cheng, Melo \& de Farias-Junior, 2014; Yao \& Rhodes, 2015), siendo esta mayor en las etapas más jóvenes y reduciéndose su influencia a medida que el niño aumenta su edad (Ryan, 2001). También se observa una pequeña influencia por parte del resto de familiares (Laird et al., 2016). De acuerdo con el estudio de Biddle et al. (2004), los resultados sugieren que el factor de auto-percepción de práctica tiene un efecto sobre la participación, al igual que los factores de percepción de salud, la competencia percibida y la participación de los padres y los compañeros, encontrándonos en el caso del factor de autopercepción de práctica con un inconveniente importante, al observarse en los resultados como los alumnos piensan que son activos, opinión que comparten sus propios padres, situación que difiere mucho de la realidad.

Los resultados obtenidos en nuestro estudio suponen un paso más en relación a los presentados en investigaciones previas en las que el análisis de la práctica de AF en adolescentes se hacía desde un punto de vista global. Esto es, el identificar las características de niñas y niños sedentarios y activos, permite la creación de perfiles que ayuden a comprender mejor el comportamiento de los adolescentes y por tanto faciliten la toma de decisiones de cara al fomento de AF en el medio escolar. Para el profesorado de educación física supone una referencia a la hora de convertir el centro educativo, en un agente promotor de estilos de vida activos y saludables, abriendo nuevas vías de actuación con el propio alumnado y con sus padres y madres.

\section{Conclusiones}

Por desgracia podemos observar como el porcentaje de jóvenes que no cumplen con los mínimos recomendados por la Organización Mundial de la Salud (2010) es muy elevado, esto supone un problema tanto para la salud de estas personas como para la economía de los países. Destacamos como dentro de la situación conflictiva, en cuanto a inactividad física nos referimos, nos encontramos al género femenino como el más perjudicado, ya que las niñas practican en menor cantidad incluso que los niños, esta situación debe estudiarse para observar

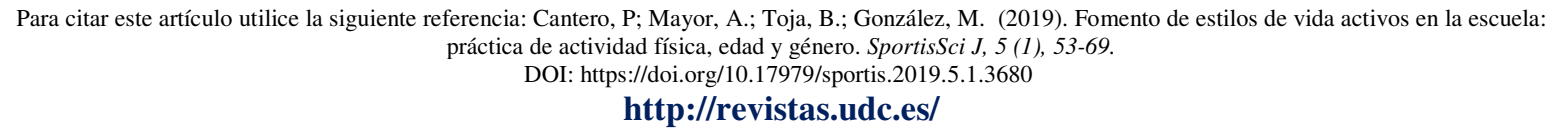




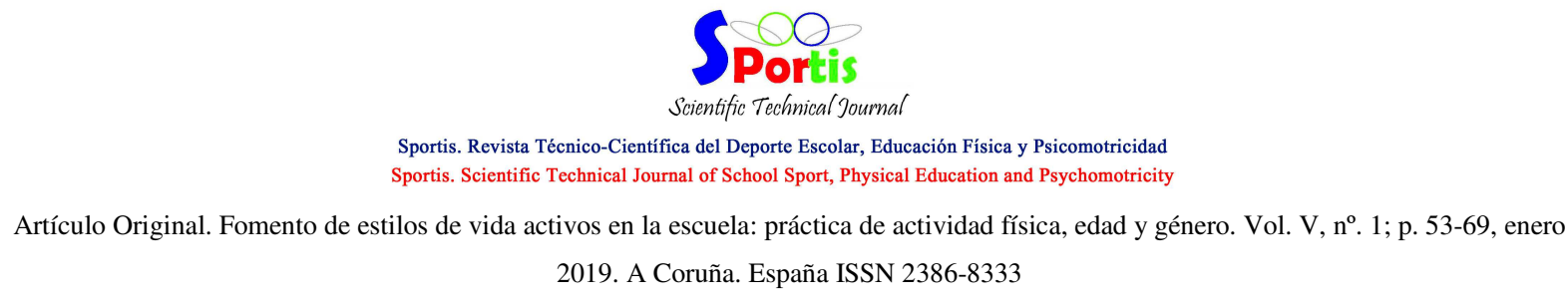

cuales son las razones que impulsan a que se produzcan estas diferencias entre género, para que así, a través de estrategias se pueda impulsar y mejorar la práctica de AF en ambos géneros, igualando en todo lo posible las diferencias entre ellos. Por lo tanto, al estudiar las percepciones que tienen los alumnos acerca de su propia práctica de $\mathrm{AF}$, nos encontramos con una gran disociación entre la percepción y la realidad, mostrándonos así un grave problema, ya que se hace más complejo a la hora de modificar una conducta como puede ser la falta de práctica si la persona no es consciente de su error y está convencido de que lo que realiza es lo correcto. A consecuencia de los resultados, se debe informar a los alumnos y a las alumnas como a sus padres y madres del verdadero problema que supone su inactividad física, para que una vez sean conscientes de ello, intenten modificar esta conducta aumentando la práctica de AF, conllevando esto una mejora de la salud. Para que esta mejora sea posible, además de concienciar a los alumnos y familiares, es importante que la oferta de práctica de AF llegue de mejor forma a estos, para que así, sea más asequible y cómodo poder optar a esta práctica.

\section{Propuestas de mejora y limitaciones del estudio}

Este estudio muestra limitaciones a consecuencia de las características del instrumento utilizado, ya que, no podemos asegurar que los niños y niñas hayan contestado sinceramente, prestando la atención necesaria a cada uno de los ítems del cuestionario. De la misma forma, aunque contamos con una muestra abundante, esta puede mostrar resultados diferentes a otras poblaciones con diferentes características, como pueden ser diferencias culturales, económicas, sociales, o de edad.

Así, se recomiendo realizar este estudio en otras comunidades autónomas y en otros rangos de edad, para comprobar si las características culturales de cada zona influyen en estos resultados, al igual que las diferencias madurativas propias de la edad.

\section{Referencias bibliográficas}

1. Aaro, L., Wold, B., Kannas, L., \& Rimpelä, M. (1986). Health Behaviour in Schoolchildren. A WHO Cross-national Survey. Health Promotion International, 1(1), 17-33.

\footnotetext{
Para citar este artículo utilice la siguiente referencia: Cantero, P; Mayor, A.; Toja, B.; González, M. (2019). Fomento de estilos de vida activos en la escuela: práctica de actividad física, edad y género. SportisSci J, 5 (1), 53-69. DOI: https://doi.org/10.17979/sportis.2019.5.1.3680

http://revistas.udc.es/
} 
2. Abarca-Sos, A., Zaragoza-Casterad, J., Generelo-Lanaspa, E., \& Julián-Clemente, J.A. (2010). Comportamientos sedentarios y patrones de actividad física en adolescentes. Revista internacional médica de ciencias de la actividad física el deporte, 10(39), 410-427.

3. Ahrabi-Fard, I., \& Matvienko, O.A. (2005). Promoción de una educación active de la actividad física orientada a la salud en las clases de Educación Física. Cultura, Ciencia y Deporte, 1(3), 163-170.

4. Arias, E.A. (2014). Niveles de actividad física de niños y adolescentes durante el descanso en la escuela, un estudio observacional con el uso de soplay. Revista Educación Física y Deporte, 33(1), 175-191.

5. Biddle, S., Gorely, T., \& Stensel, D. (2004). Health-enhancing physical activity and sedentary behaviour in children and adolescents. Journal of Sports Sciences, 22, 679-701.

6. Camacho, M.J., Fernández, E., Ramírez, E., \& Blández, J. (2012). The role of Physical Education in the promotion of health-oriented physical activity in adolescence: a systematic review of intervention programs. Revista Complutense de Educación, 24, 9-26.

7. Campbell, V.A., Crews, J.E., \& Sinclair, L. (2002). State-specific prevalence of obesity among adults with disabilities-Eight states and the District of Columbia, 1998-1999. Morbidity and Mortality Weekly Report, 51(36), 805-808.

8. Cantero, P. (2018). La percepción de salud en jóvenes escolares (Tesis Doctoral). Universidad A Coruña. A Coruña, España.

9. Cocca, A., Viciana, J., Mayorga, D., Ceballos, O., Liukkonen, J., \& Cocca, M. (2015). Adolescents and young adults physical activity related to built enviroment. Educación Física y Ciencia, 17(1).

10. Cramer, H. (1999). Mathematical methods statistical. Campaing: Princeston University Press.

11. Currie, C., Zanotti, C., Morgan, A., Currie, D., de Looze, M., Roberts, C.,...Barkenow, V. (2012). Social determinants of health and well-being among young people. Health Behaviour in School-aged Children (HSBC) study: international report from the 2009/10 survey. WHO Regional Office for Europe, 6. 
12. Ekelund, U., Luan, J., Sherar, L.B., Esliger, D.W., Griew, P., \& Cooper, A. (2012). Moderate to Vigorous Physical Activity and Sedentary Time and Cardiometabolic Risk Factors in Children and Adolescents. Journal of the American Medical Association, 307(7), 704-712.

13. Fairclough, S., \& Stratton, G. (2005). Physical education makes you fit and healthy. Physical education's contribution to young people's physical activity levels. Health Education Researh, 20(1), 14-23.

14. George, D., \& Mallery, P. (2016). IBM SPSS Statistics 23 step by step: A simple guide and reference. New York, NY: Routledge.

15. ISCA (2015). The economic cost of physical inactivity in Europe. Cebr report.

16. Janz, K.F., Dawson, J.D., \& Mahoney, L.T. (2000). Tracking physical fitness and physical activity from childhood to adolescence: the Muscatine study. Medicine and Science in Sports and Exercise, 32(7), 1250-1257.

17. Kahn, E., Ramsey, L.T., Brownson, R.C., Health, G.W., Howze, E.H., Powell, K.E.,...Corso, P. (2002). The effectiveness of interventions to increase physical activity. A systematic review. American Journal of Preventive Medicine, 22(4), 73-107.

18. Laird, Y., Fawkner, S., Kelly, P., McNamee, L., \& Niven, A. (2016). The role of social support on physical activity behaviour in adolescent girls: a systematic review and metaanalysis. International Journal of Behavioral Nutrition and Physical Activity, 13(79).

19. Marques, A. (2010). A Escola, a Educação Física e a Promoção de Estilos de Vida Activos e Saudáveis: Estudo de um Caso (Dissertação de Doutoramento). Universidade Técnica de Lisboa, Portugal.

20. Marques, A., \& Carreiro da Costa, F. (2013). Levels of Physical Activity of Urban Adolescents According to Age and Gender. International Journal of Sports Science, 3(1), 23-27.

21. Marques, A., Martins, J., Carreiro da Costa, F., \& Piéron, M. (2014): Do boys and girls share the same characteristics when equally classified as active or inactive?. Revista iberoamericana de psicología del ejercicio y el deporte, 10(2), 267-274.

\footnotetext{
Para citar este artículo utilice la siguiente referencia: Cantero, P; Mayor, A.; Toja, B.; González, M. (2019). Fomento de estilos de vida activos en la escuela: práctica de actividad física, edad y género. SportisSci J, 5 (1), 53-69. DOI: https://doi.org/10.17979/sportis.2019.5.1.3680

http://revistas.udc.es/
} 
22. Mendonça, G., Cheng, L.A., Mélo, E.N., \& de Farias-Júnior, J.C. (2014). Physical activity and social support in adolescents: a systematic review. Health Education Research, 29(5), 822-839.

23. Moral-García, J.E., Redecillas-Peiró, M.T., \& Martínez-López, E.J. (2012). Sedentary lifestyle or adolescent andalusian. Journal of Sport and Health Research, 4(1), 67-82,

24. Mota, J., \& Sallis, J. (2002) Actividade Física e Saúde. Factores de Influência da Actividade Física nas Crianças e nos Adolescentes. Porto: Campo de Letras.

25. Mourelle, M. (2014). Relación de los agentes sociales con el índice de práctica de actividad física de los escolares de 10 a 16 años de las urbes gallegas (Tesis Doctoral). Universidad A Coruña. A Coruña, España.

26. Organización Mundial de la Salud (2010). Recomendaciones mundiales sobre actividad física para la salud. Ginebra: Ediciones de la OMS.

27. Oviedo, G., Sánchez, J., Castro, R., Calvo, M., Sevilla, J.C, Iglesias, A., \& Guerra, M. (2013). Niveles de actividad física en población adolescente: estudio de caso. Retos Nuevas Tendencias en Educación Física, Deporte y Recreación, 23, 43-47.

28. Piéron, M., Telama, R., Naul, R., \& Almond, L. (1997). Etude du style de vie d'adolescents européens. Considérations théoriques, objectifs et méthodologie de recherche. Sport, 159/160, 43-50.

29. Ramos, P., Rivera, F., Moreno, C., \& Jiménez Iglesias, A. (2012). Análisis de clúster de la actividad física y las conductas sedentarias de los adolescentes españoles, correlación con la salud biopsicosocial. Revista de Psicología del Deporte, 21(1), 99-106.

30. Ryan, A. (2001). The peer group as a context for the development of young adolescent motivation and achievement. Child Development, 72, 1135-1150.

31. Sallis, J. (2000). Age-related decline in physical activity: a synthesis of human and animal studies. Medicine Science Sports Exercise, 32, 1598-1600.

32. Sallis, J.F., Bull, F., Guthold, R., Heath, G.W., Inoue, S., Kelly, P.,...Hallal, P. (2016). Progress in physical activity over the Olympic quadrennium. The Lancet, 388, 1325-1336.

33. Serra, L.L., \& Aranceta, J. (2000). Evaluación del estado nutricional y hábitos alimentarios de la población juvenil española. Estudio Enkid, (3.). Barcelona: Masson.

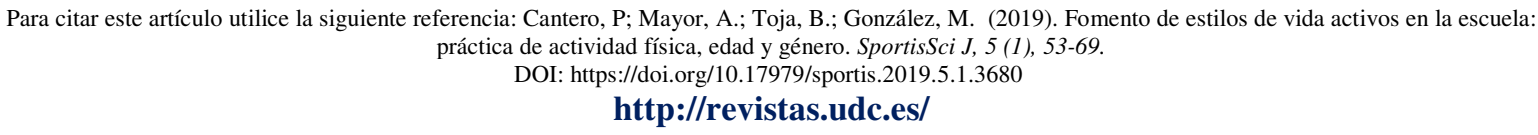


34. Telama, R., \& Yang, X. (2000). Decline of physical activity from youth to young adulthood in Finland. Medicine \& Science in Sports \& Exercise, 32, 1617-1622.

35. Telama, R., Yang, X., Laakso, L., \& Viikari. J. (1997). Physical activity in childhood and adolescence as predictor of physical activity in young adulthood. American Journal of Preventive Medicine, 13, 317-323.

36. Wold, B., \& Anderssen, N. (1992) 'Health promotion aspects of family and peer influences on sport participation', International Journal of Sport Psychology, 23, 343-359.

37. Xunta de Galicia (2013): Plan Proxecta: Proyecto Deportivo de Centro. Disponible en: http://www.edu.xunta.es/web/planproxecta.

38. Yao, C.A., \& Rhodes, R.E. (2015). Parental correlates in child and adolescent physical activity: a meta-analysis. International Journal of Behavioral Nutrition and Physical Activity, 12(10).

Para citar este artículo utilice la siguiente referencia: Cantero, P; Mayor, A.; Toja, B.; González, M. (2019). Fomento de estilos de vida activos en la escuela: práctica de actividad física, edad y género. SportisSci J, 5 (1), 53-69. 